\title{
Height of varieties over finitely generated fields
}

\author{
José Ignacio Burgos Gil, Patrice Philippon, and Martín Sombra
}

\begin{abstract}
We show that the height of a variety over a finitely generated field of characteristic zero can be written as an integral of local heights over the set of places of the field. This allows us to apply our previous work on toric varieties and extend our combinatorial formulae for the height to compute some arithmetic intersection numbers of nontoric arithmetic varieties over the rational numbers.
\end{abstract}

\section{Introduction}

Moriwaki [Mo1], [Mo2] introduced a notion of height for cycles over a finitely generated extension of $\mathbb{Q}$. With this definition, it was possible to extend several central results about cycles over a number field to cycles over a finitely generated extension of $\mathbb{Q}$. These results include Northcott's theorem on the finiteness of cycles with bounded degree and height, the Manin-Mumford and the Bogomolov conjectures, Zhang's theorem on successive algebraic minima, and the equidistribution properties of Galois orbits of points of small height (see [Mo1], [Mo2], [Mo3], [YZ]).

This notion of height is defined as follows. Let $\mathcal{B}$ be an arithmetic variety, that is, a normal flat projective scheme over $\operatorname{Spec}(\mathbb{Z})$, of relative dimension $b$. Set $\mathbb{K}=\mathrm{K}(\mathcal{B})$ for its function field, which is a finitely generated extension of $\mathbb{Q}$ of transcendence degree $b$. Let $\overline{\mathcal{H}}_{i}, i=1, \ldots, b$, be a family of nef Hermitian line bundles on $\mathcal{B}$.

Let $\pi: \mathcal{X} \rightarrow \mathcal{B}$ be a dominant morphism of arithmetic varieties, and denote by $X$ the fiber of $\pi$ over the generic point of $\mathcal{B}$, which is a variety over $\mathbb{K}$. Let $Y$ be a prime cycle of $X$ of dimension $d$. Let $\mathcal{Y}$ be the closure of $Y$ in $\mathcal{X}$, and let $\overline{\mathcal{L}}_{j}, j=0, \ldots, d$, be a family of semipositive Hermitian line bundles

Kyoto Journal of Mathematics, Vol. 56, No. 1 (2016), 13-32

DOI 10.1215/21562261-3445138, (C) 2016 by Kyoto University

Received August 15, 2014. Revised November 26, 2014. Accepted December 1, 2014.

2010 Mathematics Subject Classification: Primary 14G40; Secondary 11G50, 14M25.

Burgos Gil's work was partially supported by the Ministerio de Economía y Competitividad research project MTM2013-42135-P.

Philippon's work was partially supported by the CNRS international project for scientific cooperation (PICS) "Géométrie diophantienne et calcul formel" and the Agence Nationale de la Recherche research project "Hauteurs, modularité, transcendance."

Sombra's work was partially supported by the Ministerio de Economía y Competitividad research project MTM2012-38122-C03-02. 
on $\mathcal{X}$. Moriwaki [Mo1], [Mo2] defines the height of $Y$ relative to this data as the arithmetic intersection number in the sense of Gillet-Soule given by

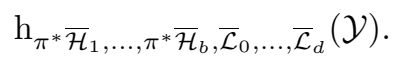

It is well known that this arithmetic intersection number can be written as a sum over the places of $\mathbb{Q}$ of local heights of the fiber of $\mathcal{Y}$ over the generic point of $\operatorname{Spec}(\mathbb{Z})$ (see, e.g., [BPS, Section 1.5]). However, for points in a projective space and the canonical metric, Moriwaki [Mo1, Proposition 3.2.2] showed that this arithmetic intersection number is also equal to an integral of local heights over a measured set of places of $\mathbb{K}$.

In this paper, we extend Moriwaki's result to a cycle $Y$ of arbitrary dimension and general semipositive metrics to show that the height of $Y$ is equal to an integral of local heights over this set of places of $\mathbb{K}$ (Theorem 2.4). This allows us to apply our previous work on toric varieties in [BPS] and extend our combinatorial formulae for the height to some arithmetic intersection numbers of nontoric arithmetic varieties. More explicitly, let $\mathcal{X} \rightarrow \mathcal{B}$ be a dominant morphism of arithmetic varieties as above such that its generic fiber $X$ is a toric variety over $\mathbb{K}$ of dimension $n$. For simplicity, suppose that $\overline{\mathcal{L}}_{0}=\cdots=\overline{\mathcal{L}}_{n}=\overline{\mathcal{L}}$. The semipositive Hermitian line bundle $\overline{\mathcal{L}}$ defines a polytope $\Delta$ in a linear space of dimension $n$ and, for each place $w$ of $\mathbb{K}$, a concave function $\vartheta_{w}: \Delta \rightarrow \mathbb{R}$ called the $w$-adic roof function (see Section 3 for details and pointers to the literature). By [BPS, Theorem 5.1.6], the $w$-adic local height of $X$ is given by $(n+1)$ ! times the integral over $\Delta$ of this concave function. Combining this with Theorem 2.4, we derive a formula for the corresponding arithmetic intersection number as an integral of the function $(w, x) \mapsto \vartheta_{w}(x)$ over the product of the polytope and the set of places of $\mathbb{K}$ (Corollary 3.1). Furthermore, we can define a global roof function $\vartheta: \Delta \rightarrow \mathbb{R}$ by integrating the local ones over the set of places of $\mathbb{K}$. Then, in this case, the arithmetic intersection number $(0.1)$ is also equal to $(n+1)$ ! times the integral of $\vartheta$ over the polytope (Corollary 3.4).

As an application, we give in Section 4 an explicit formula for the case of translates of subtori of a projective space and canonical metrics (Corollary 4.2). The obtained integrals reduce, in some instances, to logarithmic Mahler measures of multivariate polynomials.

\section{Fields with product formula from arithmetic varieties}

Gubler [G, Example 11.22] observed that, for an arithmetic variety equipped with a family of nef Hermitian line bundles, one can endow its function field with a measured set of places satisfying the product formula. In this section, we explain the details of this construction and, as an example, we explicitly describe it for the projective space and the universal line bundle equipped with the canonical metric. We refer to [BPS, Chapter 1] and [BMPS, Section 3] for the background for this section on metrized line bundles and their associated measures and heights. 
Let $\mathcal{B}$ be an arithmetic variety, which means that $\mathcal{B}$ is a normal flat projective scheme over $\operatorname{Spec}(\mathbb{Z})$. We denote by $b$ the relative dimension of $\mathcal{B}$ and by $\mathbb{K}=\mathrm{K}(\mathcal{B})$ its function field, which is a finitely generated extension of $\mathbb{Q}$ of transcendence degree $b$. For $i=1, \ldots, b$, let $\overline{\mathcal{H}}_{i}=\left(\mathcal{H}_{i},\|\cdot\|_{i}\right)$ be a Hermitian line bundle on $\mathcal{B}$, that is, a line bundle $\mathcal{H}_{i}$ on $\mathcal{B}$ equipped with a continuous metric on the complexification $\mathcal{H}_{i, \mathbb{C}}$ over $\mathcal{B}(\mathbb{C})$, invariant under complex conjugation. We will furthermore assume that each $\overline{\mathcal{H}}_{i}$ is nef in the sense of [Mo1, Section 2] and [BMPS, Definition 3.18(3)]. This amounts to the following conditions:

(1) the metric $\|\cdot\|_{i}$ is semipositive; namely, it is the uniform limit of a sequence of smooth semipositive metrics as in [Ma, Definition 4.5.5] and [BPS, Definition 1.4.1];

(2) the height of every integral one-dimensional subscheme of $\mathcal{B}$ with respect to $\overline{\mathcal{H}}_{i}$ is nonnegative.

Let $\mathcal{B}^{(1)}$ denote the set of hypersurfaces of $\mathcal{B}$, that is, the integral subschemes of $\mathcal{B}$ of codimension 1. Let $\mathcal{V} \in \mathcal{B}^{(1)}$. By [Z, Theorem 1.4(a)] or [Mo1, Proposition 2.3], the hypothesis that the $\mathcal{H}_{i}$ 's are nef implies that the height of $\mathcal{V}$ with respect to these Hermitian line bundles, denoted by $\mathrm{h}_{\overline{\mathcal{H}}_{1}, \ldots, \overline{\mathcal{H}}_{b}}(\mathcal{V})$, is nonnegative. Hence, we associate to $\mathcal{V}$ the non-Archimedean absolute value on $\mathbb{K}$ given, for $\gamma \in \mathbb{K}$, by

$$
|\gamma|_{\mathcal{V}}=\mathrm{e}^{-\mathrm{h}_{\overline{\mathcal{H}}_{1}, \ldots, \overline{\mathcal{H}}_{b}}(\mathcal{V}) \operatorname{ord} \mathcal{V}(\gamma)}
$$

where $\operatorname{ord}_{\mathcal{V}}$ denotes the discrete valuation associated to the local ring $\mathcal{O}_{\mathcal{B}, \mathcal{V}}$. We denote by $\mu_{\text {fin }}$ the counting measure of $\mathcal{B}^{(1)}$.

We define the set of generic points of $\mathcal{B}(\mathbb{C})$ as

$$
\mathcal{B}(\mathbb{C})^{\text {gen }}=\mathcal{B}(\mathbb{C}) \backslash \bigcup_{\mathcal{V} \in \mathcal{B}^{(1)}} \mathcal{V}(\mathbb{C})
$$

By definition, a point $p \in \mathcal{B}(\mathbb{C})$ belongs to $\mathcal{B}(\mathbb{C})^{\text {gen }}$ if and only if, for all $\gamma \in \mathbb{K}^{\times}$, this point does not lie in the analytification of the support of $\operatorname{div}(\gamma)$. Hence, $|\gamma(p)|$ is a well-defined positive real number, and we associate to $p$ the Archimedean absolute value given, for $\gamma \in \mathbb{K}^{\times}$, by

$$
|\gamma|_{p}=|\gamma(p)| .
$$

On $\mathcal{B}(\mathbb{C})$, we consider the measure

$$
\mu_{\infty}=\mathrm{c}_{1}\left(\overline{\mathcal{H}}_{1}\right) \wedge \cdots \wedge \mathrm{c}_{1}\left(\overline{\mathcal{H}}_{b}\right)
$$

associated to the family of semipositive Hermitian line bundles $\overline{\mathcal{H}}_{i}, i=1, \ldots, b$, as in [BPS, Definition 1.4.2]. By [CT, Corollaire 4.2], the measure of each hypersurface of $\mathcal{B}(\mathbb{C})$ with respect to $\mu_{\infty}$ is zero. Since the complement of $\mathcal{B}(\mathbb{C})^{\text {gen }}$ is a countable union of hypersurfaces, it has measure zero. We will denote also by $\mu_{\infty}$ the induced measure on $\mathcal{B}(\mathbb{C})^{\text {gen }}$.

Put then

$$
(\mathfrak{M}, \mu)=\left(\mathcal{B}^{(1)}, \mu_{\text {fin }}\right) \sqcup\left(\mathcal{B}(\mathbb{C})^{\text {gen }}, \mu_{\infty}\right)
$$


The set $\mathfrak{M}$ is in bijection with a set of absolute values. Moreover, all the nonArchimedean absolute values in this set are associated to a discrete valuation.

\section{EXAMPLE 1.1}

Let $\mathcal{B}=\mathbb{P}_{\mathbb{Z}}^{b}$ with projective coordinates $\left(x_{0}: \cdots: x_{b}\right)$, and let $\overline{\mathcal{H}}_{i}=\overline{\mathcal{O}(1)}^{\text {can }}, i=$ $1, \ldots, b$, be the universal line bundle on $\mathbb{P}_{\mathbb{Z}}^{b}$ equipped with the canonical metric as in $\left[\mathrm{BPS}\right.$, Example 1.4.4]. We have that $\mathbb{K}=\mathrm{K}(\mathcal{B}) \simeq \mathbb{Q}\left(z_{1}, \ldots, z_{b}\right)$, with $z_{i}=x_{i} / x_{0}$.

Consider the compact subtorus of $\mathbb{P}_{\mathbb{Z}}^{b}(\mathbb{C})$ given by

$$
\mathbb{S}=\left\{\left(1: z_{1}: \cdots: z_{b}\right) \in \mathbb{P}_{\mathbb{Z}}^{b}(\mathbb{C})|| z_{i} \mid=1 \text { for all } i\right\} \simeq\left(S^{1}\right)^{b}
$$

and the measure $\mu_{\mathbb{S}}$ of $\mathbb{P}^{b}(\mathbb{C})$ given by the current

$$
\frac{1}{(2 \pi i)^{b}} \frac{d z_{1}}{z_{1}} \wedge \cdots \wedge \frac{d z_{b}}{z_{b}} \wedge \delta_{\mathbb{S}}
$$

Namely, $\mu_{\mathbb{S}}$ is the Haar probability measure on $\mathbb{S}$.

A hypersurface $\mathcal{V}$ of $\mathbb{P}_{\mathbb{Z}}^{b}$ corresponds to an irreducible homogeneous polynomial $P_{\mathcal{V}} \in \mathbb{Z}\left[x_{0}, \ldots, x_{b}\right]$. The associated absolute value is given, for $\gamma \in \mathbb{K}^{\times}$, by

$$
\log |\gamma|_{\mathcal{V}}=-\operatorname{ord} \mathcal{V}(\gamma) \mathrm{m}\left(P_{\mathcal{V}}\right)
$$

where $\mathrm{m}\left(P_{\mathcal{V}}\right)$ is the logarithmic Mahler measure of $P_{\mathcal{V}}$ given by

$$
\mathrm{m}\left(P_{\mathcal{V}}\right)=\int \log \left|P_{\mathcal{V}}\left(1, z_{1}, \ldots, z_{b}\right)\right| d \mu_{\mathbb{S}}
$$

If $P_{\mathcal{V}}$ is an irreducible polynomial of degree zero, then $P_{\mathcal{V}}=p \in \mathbb{Z}$, a prime number. In this case $\mathrm{m}(P \mathcal{V})=\log (p)$ and $\mathcal{V}$ is the fiber over the point corresponding to $p$.

The absolute value associated to a point of $\mathbb{P}_{\mathbb{Z}}^{b}(\mathbb{C})^{\text {gen }}$ is given by the Archimedean absolute value of the evaluation at this point as in (1.1). In this example, the measure $\mu_{\mathrm{fin}}$ on $\left(\mathbb{P}_{\mathbb{Z}}^{b}\right)^{(1)}$ is the counting measure, and the measure $\mu_{\infty}$ is the restriction to $\mathbb{P}_{\mathbb{Z}}^{b}(\mathbb{C})^{\text {gen }}$ of $\mu_{\mathbb{S}}$.

A function on a measured space is integrable (also called summable) if its integral is a well-defined real number.

\section{PROPOSITION 1.2}

For each $\gamma \in \mathbb{K}^{\times}$, the function $\mathfrak{M} \rightarrow \mathbb{R}$ given by $w \mapsto \log |\gamma|_{w}$ is $\mu$-integrable. Furthermore, the product formula

$$
\int_{\mathfrak{M}} \log |\gamma|_{w} d \mu(w)=0
$$

holds.

Proof

Given $\gamma \in \mathbb{K}^{\times}$, the set of hypersurfaces $\mathcal{V}$ such that $|\gamma| \mathcal{V} \neq 1$ is contained in the set of components of the support of $\operatorname{div}(\gamma)$, when $\gamma$ is viewed as a rational function 
on $\mathcal{B}$. Hence, this set is finite, and so the function on $\mathcal{B}^{(1)}$ given by $\mathcal{V} \mapsto \log |\gamma| \mathcal{V}$ is

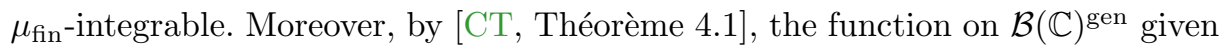
by $p \mapsto \log |\gamma(p)|$ is $\mu_{\infty}$-integrable. By summing up, $\log |\gamma|_{w}$ is $\mu$-integrable, which proves the first statement.

For the second one, let $\overline{\mathcal{O}}$ be the trivial metrized line bundle on $\mathcal{B}$. Then

$$
\begin{aligned}
\int_{\mathfrak{M}} \log |\gamma|_{w} d \mu(w) & =\sum_{\mathcal{V} \in \mathcal{B}^{(1)}}-\operatorname{ord} \mathcal{V}(\gamma) \mathrm{h}_{\overline{\mathcal{H}}_{1}, \ldots, \overline{\mathcal{H}}_{b}}(\mathcal{V})+\int_{\mathcal{B}(\mathbb{C})^{\operatorname{gen}}} \log |\gamma(p)| d \mu_{\infty}(p) \\
& =-\mathrm{h}_{\overline{\mathcal{O}}, \overline{\mathcal{H}}_{1}, \ldots, \overline{\mathcal{H}}_{b}}(\mathcal{B})
\end{aligned}
$$

by the arithmetic Bézout formula (see, e.g., [BGS, (3.2.2)] for the smooth case or [CT, Théorème 1.4] for an adelic version in the general case). From the multilinearity of the height, it follows that $\mathrm{h}_{\overline{\mathcal{O}}, \overline{\mathcal{H}}_{1}, \ldots, \overline{\mathcal{H}}_{b}}(\mathcal{B})=0$, which concludes the proof.

\section{DEFINITION 1.3}

Given $\gamma=\left(\gamma_{0}, \ldots, \gamma_{n}\right) \in \mathbb{K}^{n+1} \backslash\{\mathbf{0}\}$, the size of $\boldsymbol{\gamma}$ with respect to $(\mathbb{K}, \mathfrak{M}, \mu)$ is defined as

$$
\mathrm{t}_{\mathbb{K}, \mathfrak{M}, \mu}(\gamma)=\int_{\mathfrak{M}} \log \max \left(\left|\gamma_{0}\right|_{w}, \ldots,\left|\gamma_{n}\right|_{w}\right) d \mu(w)
$$

\section{EXAMPLE 1.4}

Let $\mathbb{K} \simeq \mathbb{Q}\left(z_{1}, \ldots, z_{b}\right)$ with the measured set of places $(\mathfrak{M}, \mu)$ as described in Example 1.1. Let $\gamma \in \mathbb{K}^{\times}$given in reduced representation as $\gamma=\alpha / \beta$ with coprime $\alpha, \beta \in \mathbb{Z}\left[z_{1}, \ldots, z_{b}\right]$. By using the product formula (1.3), the size of $\gamma$ can be given in this case by

$$
\mathrm{t}_{\mathbb{K}, \mathfrak{M}, \mu}(\gamma)=\int_{\mathfrak{M}} \log \max \left(|\alpha|_{w},|\beta|_{w}\right) d \mu(w) .
$$

Since $\alpha$ and $\beta$ are coprime, the contribution of the integral over the places of $\left(\mathbb{P}_{\mathbb{Z}}^{b}\right)^{(1)}$ is zero. Hence,

$$
\mathrm{t}_{\mathbb{K}, \mathfrak{M}, \mu}(\gamma)=\frac{1}{(2 \pi i)^{b}} \int_{\left(S^{1}\right)^{b}} \log \max (|\alpha(z)|,|\beta(z)|) \frac{d z_{1}}{z_{1}} \wedge \cdots \wedge \frac{d z_{b}}{z_{b}} .
$$

By using Jensen's formula, this size can be alternatively written as the logarithmic Mahler measure of the polynomial

$$
P_{\gamma}=\alpha\left(z_{1}, \ldots, z_{b}\right) t_{1}-\beta\left(z_{1}, \ldots, z_{b}\right) \in \mathbb{Z}\left[t_{1}, z_{1}, \ldots, z_{b}\right],
$$

where $t_{1}$ denotes an additional variable. The difference between this size and the logarithm of the maximum of the absolute values of the coefficients of $\alpha$ and $\beta$ can be bounded by the maximum of their degrees times a constant depending only on $b$. 


\section{Relative arithmetic varieties}

In this section we prove our main result (Theorem 2.4), showing that the height of a cycle over the finitely generated extension $\mathbb{K}$ can be written as an integral of the local heights of this cycle over the measured set of places $(\mathfrak{M}, \mu)$.

Let $\pi: \mathcal{X} \rightarrow \mathcal{B}$ be a dominant morphism of arithmetic varieties of relative dimension $n \geq 0$, and let $\overline{\mathcal{L}}$ be a Hermitian line bundle on $\mathcal{X}$. We denote by $X$ the fiber of $\pi$ over the generic point of $\mathcal{B}$. This is a variety over $\mathbb{K}$ of dimension $n$, and the line bundle $\mathcal{L}$ induces a line bundle on $X$, denoted by $L$. There is a collection of metrics on the analytifications of $L$ for each absolute value of $\mathfrak{M}$, which we now describe.

For each $\mathcal{V} \in \mathcal{B}^{(1)}$, the local ring $\mathcal{O}_{\mathcal{B}, \mathcal{V}}$ is a discrete valuation ring with field of fractions $\mathbb{K}$. The scheme $\mathcal{X}$ and the line bundle $\mathcal{L}$ induce a projective model over $\operatorname{Spec}\left(\mathcal{O}_{\mathcal{B}, \mathcal{V}}\right)$, denoted $\left(\mathcal{X}_{\mathcal{V}}, \mathcal{L}_{\mathcal{V}}\right)$, of the pair $(X, L)$. Following Zhang, we see that the model $\left(\mathcal{X}_{\mathcal{V}}, \mathcal{L}_{\mathcal{V}}\right)$ induces a metric on the analytification $L_{\mathcal{V}}^{\text {an }}$ over $X_{\mathcal{V}}^{\text {an }}$ (see [Z] or [BPS, Definition 1.3.5] for details).

The map $\pi$ also induces a map of complex analytic spaces $\mathcal{X}(\mathbb{C}) \rightarrow \mathcal{B}(\mathbb{C})$, which we also denote by $\pi$. A point $p \in \mathcal{B}(\mathbb{C})^{\text {gen }}$ induces an Archimedean absolute value $|\cdot|_{p}$ on $\mathbb{K}$, and the analytification of the variety $X$ with respect to $|\cdot|_{p}$ can be identified with the fiber $\pi^{-1}(p) \subset \mathcal{X}(\mathbb{C})$, with its structure of real analytic space when the point $p$ is real. The analytification of the line bundle $L$ on $X$ with respect to $|\cdot|_{p}$ can also be identified with the restriction of $\mathcal{L}_{\mathbb{C}}$ to $\pi^{-1}(p)$. Then the metric on $L_{p}^{\text {an }}$ is defined as the restriction of the metric on $\mathcal{L}_{\mathbb{C}}$ to this fiber. We then denote the obtained $\mathfrak{M}$-metrized line bundle on $X$ by

$$
\bar{L}=\left(L,\left(\|\cdot\|_{w}\right)_{w \in \mathfrak{M}}\right) .
$$

Let $Y$ be a $d$-dimensional cycle on $X$, and let $\bar{L}_{i}, i=0, \ldots, d$, be $\mathfrak{M}$-metrized line bundles on $X$ as in (2.1). We assume that each $\bar{L}_{i}$ is constructed from a difference of semipositive (DSP) Hermitian line bundle $\overline{\mathcal{L}}_{i}$ on $\mathcal{X}$. Recall that a DSP Hermitian line bundle on $\mathcal{X}$ is the quotient of two semipositive ones as in [BPS, Definition 1.4.1].

Given a collection of nonzero rational sections $s_{i}$ of $\mathcal{L}_{i}, i=0, \ldots, d$, intersecting properly on $Y$ and $w \in \mathfrak{M}$, we denote by

$$
\mathrm{h}_{\bar{L}_{0, w}, \ldots, \bar{L}_{d, w}}\left(Y ; s_{0}, \ldots, s_{d}\right)
$$

the local height of $Y$ with respect to the family of $w$-adic metrized line bundles $\bar{L}_{i, w}:=\left(L_{i},\|\cdot\|_{i, w}\right), i=0, \ldots, d$. It is defined inductively on the dimension of $Y$ by the arithmetic Bézout formula (see [BPS, Definition 1.4.11])

$$
\begin{aligned}
\mathrm{h}_{\bar{L}_{0, w}, \ldots, \bar{L}_{d, w}}\left(Y ; s_{0}, \ldots, s_{d}\right)= & \mathrm{h}_{\bar{L}_{0, w}, \ldots, \bar{L}_{d, w}}\left(Y \cdot \operatorname{div} s_{d} ; s_{0}, \ldots, s_{d-1}\right) \\
& -\int_{X_{w}^{\text {an }}} \log \left\|s_{d}\right\|_{d, w} \bigwedge_{i=0}^{d-1} \mathrm{c}_{1}\left(\bar{L}_{i, w}\right) \wedge \delta_{Y} .
\end{aligned}
$$


We will show in Theorem 2.4 below that the function $\mathfrak{M} \rightarrow \mathbb{R}$ given, for $w \in \mathfrak{M}$, by

$$
w \longmapsto \mathrm{h}_{\left(L_{0},\|\cdot\|_{0, w}\right), \ldots,\left(L_{d},\|\cdot\|_{d, w}\right)}\left(Y ; s_{0}, \ldots, s_{d}\right)
$$

is $\mu$-integrable.

\section{DEFINITION 2.1}

With notation as above, the global height of $Y$ with respect to the $\mathfrak{M}$-metrized line bundles $\bar{L}_{i}, i=0, \ldots, d$, is defined as the integral of the function in (2.3); that is,

$$
\mathrm{h}_{\bar{L}_{0}, \ldots, \bar{L}_{d}}(Y)=\int_{\mathfrak{M}} \mathrm{h}_{\left(L_{0},\|\cdot\|_{0, w}\right), \ldots,\left(L_{d},\|\cdot\|_{d, w}\right)}\left(Y ; s_{0}, \ldots, s_{d}\right) d \mu(w) .
$$

Thanks to the product formula, this notion does not depend on the choice of the sections $s_{i}$.

\section{EXAMPLE 2.2}

Let $\mathcal{B}$ be an arithmetic variety as above, and $(\mathbb{K}, \mathfrak{M}, \mu)$ the associated finitely generated field and measured set of places. Let

$$
\mathcal{X}=\mathbb{P}_{\mathcal{B}}^{n} \simeq \mathcal{B} \times \mathbb{P}_{\mathbb{Z}}^{n} \quad \text { and } \quad \overline{\mathcal{L}}=\varpi^{*}{\overline{\mathcal{O}_{\mathbb{P}_{\mathbb{Z}}^{n}}}}^{\text {can }}
$$

where $\varpi$ denotes the projection $\mathcal{B} \times \mathbb{P}_{\mathbb{Z}}^{n} \rightarrow \mathbb{P}_{\mathbb{Z}}^{n}$. Hence, $X=\mathbb{P}_{\mathbb{K}}^{n}$ and $\bar{L}={\overline{\mathcal{O}_{\mathbb{P}_{\mathbb{K}}^{n}}}}^{\text {can }}$. For a point $p=\left(\gamma_{0}: \cdots: \gamma_{n}\right) \in X(\mathbb{K})=\mathbb{P}_{\mathbb{K}}^{n}(\mathbb{K})$, we have

$$
\mathrm{h}_{\bar{L}}(p)=\mathrm{t}_{\mathbb{K}, \mathfrak{M}, \mu}(\gamma)
$$

where $\mathrm{t}_{\mathbb{K}, \mathfrak{M}, \mu}(\boldsymbol{\gamma})$ denotes the size of the vector $\gamma=\left(\gamma_{0}, \ldots, \gamma_{n}\right)$ as in (1.4). This is the "naive height" in [Mo1, Section 3.2].

The following projection formula for heights of schemes over $\operatorname{Spec}(\mathbb{Z})$ generalizes [Mo1, Proposition 1.3(1)].

\section{PROPOSITION 2.3}

Let $\pi: \mathcal{W} \rightarrow \mathcal{V}$ be a morphism between two finitely generated projective schemes over $\operatorname{Spec}(\mathbb{Z})$ of relative dimensions $d+b-1$ and $b-1$, respectively, with $b, d \geq 0$. Let $\overline{\mathcal{L}}_{i}, i=1, \ldots, d$, and $\overline{\mathcal{H}}_{j}, j=1, \ldots, b$, be DSP Hermitian line bundles on $\mathcal{W}$ and $\mathcal{V}$, respectively. Then

$$
\mathrm{h}_{\pi^{*} \overline{\mathcal{H}}_{1}, \ldots, \pi * \overline{\mathcal{H}}_{b}, \overline{\mathcal{L}}_{1}, \ldots, \overline{\mathcal{L}}_{d}}(\mathcal{W})=\operatorname{deg}_{\mathcal{L}_{1}, \ldots, \mathcal{L}_{d}}\left(\mathcal{W}_{\eta}\right) \mathrm{h}_{\overline{\mathcal{H}}_{1}, \ldots, \overline{\mathcal{H}}_{b}}(\mathcal{V}),
$$

where $\mathcal{W}_{\eta}$ denotes the fiber of $\mathcal{W}$ over the generic point $\eta$ of $\mathcal{V}$. In particular, if $\pi$ is not dominant, then $\mathrm{h}_{\pi * \overline{\mathcal{H}}_{1}, \ldots, \pi * \overline{\mathcal{H}}_{b}, \overline{\mathcal{L}}_{1}, \ldots, \overline{\mathcal{L}}_{d}}(\mathcal{W})=0$.

\section{Proof}

By linearity, we can reduce to the case when the $\mathcal{L}_{i}$ 's are ample and the metrics are semipositive. By continuity, we can also reduce to the case when the metrics in $\overline{\mathcal{L}_{i}}$ and $\overline{\mathcal{H}}_{j}$ are smooth for all $i, j$. 
We proceed by induction on $d$. The case $d=0$ is given by [Mo1, Proposition 1.3(2)] in the case when $\pi$ is dominant and by [BPS, Theorem 1.5.11(2)] in the general case. Let $d \geq 1$, and choose a nonzero rational section $s_{d}$ of $\mathcal{L}_{d}$. Let $\|\cdot\|_{d}$ denote the metric of $\overline{\mathcal{L}_{d}}$. By the arithmetic Bézout formula,

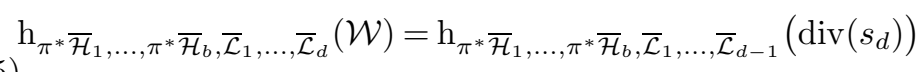

$$
-\int_{\mathcal{W}(\mathbb{C})} \log \left\|s_{d}\right\|_{d} \bigwedge_{i=1}^{b} \mathrm{c}_{1}\left(\pi^{*} \overline{\mathcal{H}}_{i}\right) \wedge \bigwedge_{j=1}^{d-1} \mathrm{c}_{1}\left(\overline{\mathcal{L}}_{j}\right) .
$$

Since $\operatorname{dim}(\mathcal{V}(\mathbb{C}))=b-1$, we have that $\bigwedge_{i=1}^{b} \mathrm{c}_{1}\left(\overline{\mathcal{H}}_{i}\right)=0$. Hence, the measure in the integral on the right-hand side of $(2.5)$ is zero, so this integral is zero too. Decompose the divisor of $s_{d}$ into its horizontal and vertical components over $\operatorname{Spec}(\mathbb{Z})$ as

$$
\operatorname{div}\left(s_{d}\right)=\operatorname{div}\left(s_{d}\right)_{\text {hor }}+\operatorname{div}\left(s_{d}\right)_{\text {vert }} .
$$

Write $\operatorname{div}\left(s_{d}\right)_{\text {vert }}=\sum_{\mathfrak{p} \in \operatorname{Spec}(\mathbb{Z})} Z_{\mathfrak{p}}$ as a finite sum of schemes over the primes. We have that $\operatorname{deg}_{\pi^{*} \mathcal{H}_{1}, \ldots, \pi^{*} \mathcal{H}_{b}, \mathcal{L}_{1}, \ldots, \mathcal{L}_{d-1}}\left(Z_{\mathfrak{p}}\right)=0$ because $\operatorname{dim}\left(\pi\left(\operatorname{div}\left(s_{d}\right)_{\text {vert }}\right)\right) \leq b-1$. It follows that

$$
\begin{aligned}
\mathrm{h}_{\pi} * \overline{\mathcal{H}}_{1}, \ldots, \pi^{*} \overline{\mathcal{H}}_{b}, \overline{\mathcal{L}}_{1}, \ldots, \overline{\mathcal{L}}_{d-1} & \left(\operatorname{div}\left(s_{d}\right)_{\text {vert }}\right) \\
\quad & =\sum_{\mathfrak{p} \in \operatorname{Spec}(\mathbb{Z})} \log (\mathfrak{p}) \operatorname{deg}_{\pi * \mathcal{H}_{1}, \ldots, \pi^{*} \mathcal{H}_{b}, \mathcal{L}_{1}, \ldots, \mathcal{L}_{d-1}}\left(Z_{\mathfrak{p}}\right)=0 .
\end{aligned}
$$

By the inductive hypothesis,

$$
\mathrm{h}_{\pi^{*} \overline{\mathcal{H}}_{1}, \ldots, \pi^{*} \overline{\mathcal{H}}_{b}, \overline{\mathcal{L}}_{1}, \ldots, \overline{\mathcal{L}}_{d-1}}\left(\operatorname{div}\left(s_{d}\right)_{\text {hor }}\right)=\operatorname{deg}_{\mathcal{L}_{1}, \ldots, \mathcal{L}_{d-1}}\left(\operatorname{div}\left(s_{d}\right)_{\text {hor }, \eta}\right) \mathrm{h}_{\overline{\mathcal{H}}_{1}, \ldots, \overline{\mathcal{H}}_{b}}(\mathcal{V}) .
$$

Since $\operatorname{deg}_{\mathcal{L}_{1}, \ldots, \mathcal{L}_{d-1}}\left(\operatorname{div}\left(s_{d}\right)_{\text {hor }, \eta}\right)=\operatorname{deg}_{\mathcal{L}_{1}, \ldots, \mathcal{L}_{d}}\left(\mathcal{W}_{\eta}\right)$, we obtain the result.

\section{THEOREM 2.4}

Let $\mathcal{B}$ be an arithmetic variety of relative dimension $b$, and $\overline{\mathcal{H}}_{i}, i=1, \ldots, b$, nef Hermitian line bundles on $\mathcal{B}$. Let $\mathbb{K}=\mathrm{K}(\mathcal{B})$ be the function field of $\mathcal{B}$, and let $(\mathfrak{M}, \mu)$ be the associated measured set of places as in (1.2).

Let $\pi: \mathcal{X} \rightarrow \mathcal{B}$ be a dominant morphism of arithmetic varieties of relative dimension $n$, and $X$ the fiber of $\pi$ over the generic point of $\mathcal{B}$. Let $Y$ be a prime cycle of $X$ of dimension $d$, and $\mathcal{Y}$ its closure in $\mathcal{X}$. Let $\overline{\mathcal{L}}_{j}, j=0, \ldots, d$, be DSP Hermitian line bundles on $\mathcal{X}$, and $\bar{L}_{j}, j=0, \ldots, d$, the associated $\mathfrak{M}$ metrized line bundles as in (2.1). Let $s_{0}, \ldots, s_{d}$ be rational sections of $\mathcal{L}_{0}, \ldots, \mathcal{L}_{d}$, respectively, intersecting properly on $Y$. Then the function $\mathfrak{M} \rightarrow \mathbb{R}$ given, for $w \in \mathfrak{M}$, by

$$
w \longmapsto \mathrm{h}_{\left(L_{0},\|\cdot\|_{0, w}\right), \ldots,\left(L_{d},\|\cdot\|_{d, w}\right)}\left(Y ; s_{0}, \ldots, s_{d}\right)
$$

is $\mu$-integrable. Moreover,

$$
\mathrm{h}_{\bar{L}_{0}, \ldots, \bar{L}_{d}}(Y)=\mathrm{h}_{\pi * \overline{\mathcal{H}}_{1}, \ldots, \pi * \overline{\mathcal{H}}_{b}, \overline{\mathcal{L}}_{0}, \ldots, \overline{\mathcal{L}}_{d}}(\mathcal{Y}) .
$$


In other words, the integral of the function (2.6) coincides with the height of $Y$ as defined in (0.1).

\section{Proof}

By linearity, we reduce to the case when the line bundles $\mathcal{L}_{j}$ are ample, their metrics are semipositive, and the sections are global sections. Moreover, since multiplying one of the metrics on $\mathcal{L}_{j}$ changes both sides of the equality (2.7) by the same additive constant, we can assume that the sections $s_{j}$ of $\mathcal{L}_{j}, j=0, \ldots, d$, are small, in the sense that $\sup _{p \in X(\mathbb{C})}\left\|s_{j}(p)\right\|_{j} \leq 1$.

We proceed by induction on the dimension of $Y$. If $\operatorname{dim}(Y)=-1$, then $Y=\emptyset$ and so the local heights of $Y$ are zero. Hence, these local heights are $\mu$-integrable and, by Proposition 2.3, the equality in (2.7) is reduced to $0=0$.

We now assume that $\operatorname{dim}(Y)=d \geq 0$. In this case, the restriction $\left.\pi\right|_{\mathcal{Y}}: \mathcal{Y} \rightarrow \mathcal{B}$ is dominant. Since the height does not change by normalization, by restricting objects to $\mathcal{Y}$ and pulling back to its normalization, we may assume in the computations that follow that $\mathcal{Y}=\mathcal{X}$. In particular, $Y=X$ and $d=n=\operatorname{dim}(X)$.

Let $s_{0}, \ldots, s_{n}$ be global sections of $\mathcal{L}_{0}, \ldots, \mathcal{L}_{n}$, respectively, that meet properly on $X$, and denote by $\rho: \mathfrak{M} \rightarrow \mathbb{R}$ the local height function in (2.6). We have to show that this function is $\mu$-integrable and that

$$
\int_{\mathfrak{M}} \rho(w) d \mu(w)=\mathrm{h}_{\pi^{*} \overline{\mathcal{H}}_{1}, \ldots, \pi^{*} \overline{\mathcal{H}}_{b}, \overline{\mathcal{L}}_{0}, \ldots, \overline{\mathcal{L}}_{n}}(\mathcal{X}) .
$$

For each $w \in \mathfrak{M}$, by the definition of local heights in (2.2), we can write $\rho(w)=\rho_{1}(w)-\rho_{2}(w)$ with

$$
\begin{aligned}
& \rho_{1}(w)=\mathrm{h}_{\left(L_{0},\|\cdot\|_{0, w}\right), \ldots,\left(L_{n-1},\|\cdot\|_{n-1, w}\right)}\left(\operatorname{div}\left(s_{n}\right) ; s_{0}, \ldots, s_{n-1}\right), \\
& \rho_{2}(w)=\int_{X_{w}^{\operatorname{an}}} \log \left\|s_{n}\right\|_{n, w} \bigwedge_{j=0}^{n-1} \mathrm{c}_{1}\left(L_{j},\|\cdot\|_{j, w}\right) .
\end{aligned}
$$

We decompose the cycle $\operatorname{div}\left(s_{n}\right)$ as

$$
\operatorname{div}\left(s_{n}\right)=\operatorname{div}\left(s_{n}\right)_{\text {hor } / \mathcal{B}}+\operatorname{div}\left(s_{n}\right)_{\operatorname{vert} / \mathcal{B}},
$$

where $\operatorname{div}\left(s_{n}\right)_{\text {hor } / \mathcal{B}}$ contains all the components that are dominant over $\mathcal{B}$ and $\operatorname{div}\left(s_{n}\right)_{\text {vert } / \mathcal{B}}$ contains the remaining ones. Clearly, $\operatorname{div}\left(s_{n}\right)_{\text {hor } / \mathcal{B}}$ is the closure of $\operatorname{div}\left(s_{n}\right) \cdot X$, and $\operatorname{div}\left(s_{n}\right)_{\text {vert } / \mathcal{B}}$ contains all the components of $\operatorname{div}\left(s_{n}\right)$ that do not meet $X$.

By the inductive hypothesis, the function $w \mapsto \rho_{1}(w)$ is $\mu$-integrable and

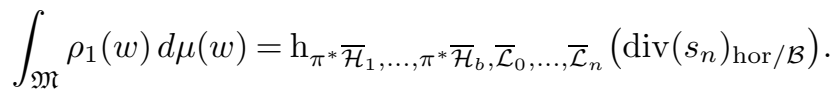

Let now $w=\mathcal{V} \in \mathcal{B}^{(1)}$. The local $\operatorname{ring} \mathcal{O}_{\mathcal{B}, \mathcal{V}}$ is a discrete valuation ring. The scheme $\mathcal{X}$ and the line bundle $\mathcal{L}_{i}$ induce models $\mathcal{X}_{\mathcal{V}}$ and $\mathcal{L}_{i, \mathcal{V}}$ over $\operatorname{Spec}\left(\mathcal{O}_{\mathcal{B}, \mathcal{V}}\right)$ of $X$ and $L_{i}$. Each component of the special fiber of $\mathcal{X}_{\mathcal{V}}$ is the localization

$$
\mathcal{W}_{\mathcal{V}}=\mathcal{W} \underset{\mathcal{V}}{\times} \operatorname{Spec}(K(\mathcal{V}))
$$


of a hypersurface $\mathcal{W} \in \mathcal{X}^{(1)}$ with $\pi(\mathcal{W})=\mathcal{V}$. Since the metric over $w$ is an algebraic metric coming from a model, by [BPS, (1.3.6) and Remark 1.4.14],

$$
\rho_{2}(\mathcal{V})=-\sum_{\substack{\mathcal{W} \in \mathcal{X}^{(1)} \\ \pi(\mathcal{W})=\mathcal{V}}} \mathrm{h}_{\overline{\mathcal{H}}_{1}, \ldots, \overline{\mathcal{H}}_{b}}(\mathcal{V}) \operatorname{ord}_{\mathcal{W}}\left(s_{n}\right) \operatorname{deg}_{\mathcal{L}_{0}, \ldots, \mathcal{L}_{n-1}}\left(\mathcal{W}_{\mathcal{V}}\right)
$$

Since the number of components of $\operatorname{div}\left(s_{n}\right)$ is finite, we deduce from (2.9) that there are only a finite number of $\mathcal{V} \in \mathcal{B}^{(1)}$ with $\rho_{2}(\mathcal{V}) \neq 0$. Thus, $\rho_{2}$ is integrable on $\mathcal{B}^{(1)}$ with respect to the counting measure $\mu_{\text {fin }}$ as in (1.2). By Proposition 2.3,

$$
\mathrm{h}_{\overline{\mathcal{H}}_{1}, \ldots, \overline{\mathcal{H}}_{b}}(\mathcal{V}) \operatorname{deg}_{\mathcal{L}_{0}, \ldots, \mathcal{L}_{n-1}}\left(\mathcal{W}_{\mathcal{V}}\right)=\mathrm{h}_{\pi^{*} \overline{\mathcal{H}}_{1}, \ldots, \pi * \overline{\mathcal{H}}_{b}, \overline{\mathcal{L}}_{0}, \ldots, \overline{\mathcal{L}}_{n-1}}(\mathcal{W})
$$

The same result implies that if $\operatorname{dim}(\pi(\mathcal{W})) \leq b-1$, then

$$
\mathrm{h}_{\pi * \overline{\mathcal{H}}_{1}, \ldots, \pi * \overline{\mathcal{H}}_{b}, \overline{\mathcal{L}}_{0}, \ldots, \overline{\mathcal{L}}_{n-1}}(\mathcal{W})=0 .
$$

Since

$$
\begin{aligned}
\operatorname{div}\left(s_{n}\right)_{\text {vert } / \mathcal{B}} & =\sum_{\substack{\mathcal{W} \in \mathcal{X}^{(1)} \\
\operatorname{dim}(\pi(\mathcal{W})) \leq b}} \operatorname{ord}_{\mathcal{W}}\left(s_{n}\right) \mathcal{W} \\
& =\sum_{\mathcal{V} \in \mathcal{B}^{(1)}} \sum_{\substack{\mathcal{W} \in \mathcal{X}^{(1)} \\
\pi(\mathcal{W})=\mathcal{V}}} \operatorname{ord}_{\mathcal{W}}\left(s_{n}\right) \mathcal{W}+\sum_{\substack{\mathcal{W} \in \mathcal{X}^{(1)} \\
\operatorname{dim}(\pi(\mathcal{W})) \leq b-1}} \operatorname{ord}_{\mathcal{W}}\left(s_{n}\right) \mathcal{W},
\end{aligned}
$$

it follows from (2.9) that

$$
\begin{aligned}
\int_{\mathcal{B}^{(1)}} \rho_{2}(w) d \mu_{\text {fin }}(w) & =\sum_{\mathcal{V} \in \mathcal{B}^{(1)}} \rho_{2}(\mathcal{V}) \\
& =-\sum_{\mathcal{V} \in \mathcal{B}^{(1)}} \sum_{\substack{\mathcal{W} \in \mathcal{X}^{(1)} \\
\pi(\mathcal{W})=\mathcal{V}}} \operatorname{ord}_{\mathcal{W}}\left(s_{n}\right) \mathrm{h}_{\pi^{*} \overline{\mathcal{H}}_{1}, \ldots, \pi^{*} \overline{\mathcal{H}}_{b}, \overline{\mathcal{L}}_{0}, \ldots, \overline{\mathcal{L}}_{n}}(\mathcal{W}) \\
& =-\mathrm{h}_{\pi^{*} \overline{\mathcal{H}}_{1}, \ldots, \pi^{*} \overline{\mathcal{H}}_{b}, \overline{\mathcal{L}}_{0}, \ldots, \overline{\mathcal{L}}_{n}}\left(\operatorname{div}\left(s_{n}\right)_{\text {vert } / \mathcal{B}}\right) .
\end{aligned}
$$

We next consider the places associated to the points $p \in \mathcal{B}(\mathbb{C})^{\text {gen }}$. In this case, by the definition of $\rho_{2}$, we have that

$$
\rho_{2}(p)=\int_{\pi^{-1}(p)} \log \left\|s_{n}\right\|_{n} \bigwedge_{j=0}^{n-1} \mathrm{c}_{1}\left(\left.\overline{\mathcal{L}}_{j, \mathbb{C}}\right|_{\pi^{-1}(p)}\right),
$$

where $\pi$ denotes the projection $\mathcal{X} \rightarrow \mathcal{B}$ and $\|\cdot\|_{n}$ the metric in $\overline{\mathcal{L}}_{n}$. We have to show that $\rho_{2}$ is $\mu_{\infty}$-integrable with $\mu_{\infty}=\bigwedge_{i=1}^{b} c_{1}\left(\overline{\mathcal{H}}_{i}\right)$ and that

$$
\int_{\mathcal{B}(\mathbb{C})^{\operatorname{gen}}} \rho_{2}(p) d \mu_{\infty}(p)=\int_{\mathcal{X}(\mathbb{C})} \log \left\|s_{n}\right\|_{n} \bigwedge_{j=0}^{n-1} \mathrm{c}_{1}\left(\overline{\mathcal{L}}_{j}\right) \wedge \bigwedge_{i=1}^{b} \mathrm{c}_{1}\left(\pi^{*} \overline{\mathcal{H}}_{i}\right) .
$$

We first assume that, for each $j=0, \ldots, n$, the metric on the line bundle $\mathcal{L}_{j}$ is smooth, but that the metric on $\mathcal{H}_{i}, i=1, \ldots, b$, is not necessarily smooth. By definition, there is a sequence of smooth semipositive metrics $\left(\|\cdot\|_{i, k}\right)_{k \geq 0}$ on $\mathcal{H}_{i, \mathbb{C}}$ that converge to $\|\cdot\|_{i}$. Set $\overline{\mathcal{H}}_{i, k}=\left(\mathcal{H}_{i},\|\cdot\|_{i, k}\right)$, and let $\mu_{\infty, k}$ be the measure 
associated to the differential form

$$
\mathrm{c}_{1}\left(\overline{\mathcal{H}}_{1, k}\right) \wedge \cdots \wedge \mathrm{c}_{1}\left(\overline{\mathcal{H}}_{b, k}\right) .
$$

By [CT, Théorème 4.1], the measures $\mu_{\infty, k}$ converge weakly to $\mu_{\infty}$. By the same result, even if $\log \left\|s_{n}\right\|_{n}$ is not bounded, the equality

$$
\begin{gathered}
\lim _{k \rightarrow \infty} \int_{\mathcal{X}(\mathbb{C})} \log \left\|s_{n}\right\|_{n} \bigwedge_{j=0}^{n-1} c_{1}\left(\overline{\mathcal{L}}_{j}\right) \wedge \bigwedge_{i=1}^{b} c_{1}\left(\pi^{*} \overline{\mathcal{H}}_{i, k}\right) \\
\quad=\int_{\mathcal{X}(\mathbb{C})} \log \left\|s_{n}\right\|_{n} \bigwedge_{j=0}^{n-1} c_{1}\left(\overline{\mathcal{L}}_{j}\right) \wedge \bigwedge_{i=1}^{b} c_{1}\left(\pi^{*} \overline{\mathcal{H}}_{i}\right)
\end{gathered}
$$

holds. Let $U \subset \mathcal{B}(\mathbb{C})$ be a connected Zariski-open subset such that the restriction $\left.\pi\right|_{\pi^{-1}(U)}$ is a proper smooth map. By Ehresmann's fibration theorem, this restriction is a locally trivial proper differentiable fibration. Thus, there exist a compact differentiable manifold $F$ and an analytic open cover $\left\{U_{\alpha}\right\}_{\alpha}$ of $U$ such that $\pi^{-1}\left(U_{\alpha}\right)$ is diffeomorphic to $F \times U_{\alpha}$ for every $\alpha$. Let $\left\{\nu_{\alpha}\right\}_{\alpha}$ be a partition of unity subordinated to the open cover $\left\{U_{\alpha}\right\}_{\alpha}$.

Fix an $\alpha$. To avoid burdening the notation, we identify $\pi^{-1}\left(U_{\alpha}\right)$ with $F \times U_{\alpha}$ through the above diffeomorphism. Let $\lambda_{F}$ be a measure of $F$ given by a volume form. Since the metrics $\|\cdot\|_{j}$ are smooth, there is a smooth function $g: F \times U_{\alpha} \rightarrow$ $\mathbb{R}$ such that, for each $u \in U_{\alpha}$,

$$
\left.\bigwedge_{j=0}^{n-1} \mathrm{c}_{1}\left(\overline{\mathcal{L}}_{j}\right)\right|_{\{u\} \times F}=g(\cdot, u) \lambda_{F} .
$$

By [B, Theorem 3.2], the measures $\lambda_{F} \times \bigwedge_{i=1}^{b} \mathrm{c}_{1}\left(\pi^{*} \overline{\mathcal{H}}_{i, k}\right)$ converge weakly to the measure $\lambda_{F} \times \bigwedge_{i=1}^{b} \mathrm{c}_{1}\left(\pi^{*} \overline{\mathcal{H}}_{i}\right)$. By the unicity of weak limits of measures,

$$
\left.\bigwedge_{j=0}^{n-1} \mathrm{c}_{1}\left(\overline{\mathcal{L}}_{j}\right) \wedge \bigwedge_{i=1}^{b} \mathrm{c}_{1}\left(\pi^{*} \overline{\mathcal{H}}_{i}\right)\right|_{F \times U_{\alpha}}=g \lambda_{F} \times \bigwedge_{i=1}^{b} \mathrm{c}_{1}\left(\pi^{*} \overline{\mathcal{H}}_{i}\right) .
$$

Since $\log \left\|s_{n}\right\|_{n}$ is integrable with respect to $\bigwedge_{j=0}^{n-1} \mathrm{c}_{1}\left(\overline{\mathcal{L}}_{j}\right) \wedge \bigwedge_{i=1}^{b} \mathrm{c}_{1}\left(\pi^{*} \overline{\mathcal{H}}_{i}\right)$, by (2.12) the function $\left(\nu_{\alpha} \circ \pi\right) \log \left\|s_{n}\right\|_{n} g$ is integrable with respect to $\lambda_{F} \times$ $\bigwedge_{i=1}^{b} \mathrm{c}_{1}\left(\pi^{*} \overline{\mathcal{H}}_{i}\right)$. By Fubini's theorem [F, Theorem 2.6.2], the function

$$
\int_{F}\left(\nu_{\alpha} \circ \pi\right) \log \left\|s_{n}\right\|_{n} g \lambda_{F}=\nu_{\alpha} \rho_{2}
$$

is $\mu_{\infty}$-integrable and

$$
\begin{aligned}
\int_{U_{\alpha}} \nu_{\alpha} \rho_{2}(p) d \mu_{\infty}(p) & =\int_{F \times U_{\alpha}}\left(\nu_{\alpha} \circ \pi\right) \log \left\|s_{n}\right\|_{n} g \lambda_{F} \times \bigwedge_{i=1}^{b} c_{1}\left(\pi^{*} \overline{\mathcal{H}}_{i}\right) \\
& =\int_{\pi^{-1}\left(U_{\alpha}\right)}\left(\nu_{\alpha} \circ \pi\right) \log \left\|s_{n}\right\|_{n} \bigwedge_{j=0}^{n-1} c_{1}\left(\overline{\mathcal{L}}_{j}\right) \wedge \bigwedge_{i=1}^{b} c_{1}\left(\pi^{*} \overline{\mathcal{H}}_{i}\right),
\end{aligned}
$$

where the last equality follows from (2.12). 
Since the above holds for every $\alpha$, Lebesgue's monotone convergence theorem [F, Corollary 2.4.8] and the fact that $\mu_{\infty}(\mathcal{B}(\mathbb{C}) \backslash U)=0$, which follows from [CT, Corollaire 4.2], imply that $\rho_{2}$ is $\mu_{\infty}$-integrable and that (2.11) holds. Observe that we can apply Lebesgue's monotone convergence theorem because we are assuming that the section $s_{n}$ is small, so the function $\log \left\|s_{n}\right\|_{n}$ is nonpositive.

We now assume that the metrics on $\mathcal{L}_{j}$ and $\mathcal{H}_{i}$ are not necessarily smooth, and we choose sequences of smooth semipositive metrics $\left(\|\cdot\|_{j, k_{j}}\right)_{k_{j} \geq 0}$ on $\mathcal{L}_{j}$ that converge uniformly to $\|\cdot\|_{j}$. For $p \in \mathcal{B}(\mathbb{C})^{\text {gen }}$, write

$$
\rho_{2, k_{0}, \ldots, k_{n}}(p)=\int_{\pi^{-1}(p)} \log \left\|s_{n}(p)\right\|_{n, k_{n}} \bigwedge_{j=0}^{n-1} \mathrm{c}_{1}\left(\left.\overline{\mathcal{L}}_{j, k_{j}, \mathbb{C}}\right|_{\pi^{-1}(p)}\right) .
$$

From (2.13) when $i=n$ and from (2.13) and Stokes's theorem when $i \neq n$, one can prove that, for each $\varepsilon>0$, there is a constant $K_{i}$ that does not depend on $p$ nor on $k_{j}, j \neq i$, such that, for all $k_{i}, k_{i}^{\prime} \geq K_{i}$,

$$
\left|\rho_{2, k_{0}, \ldots, k_{i}, \ldots, k_{n}}(p)-\rho_{2, k_{0}, \ldots, k_{i}^{\prime}, \ldots, k_{n}}(p)\right| \leq \varepsilon .
$$

For $k \geq 0$, denote by $\rho_{2, k}$ the function in (2.13) for the choice of indices $k_{0}=\cdots=k_{n}=k$. We deduce from (2.14) that the diagonal sequence $\left(\rho_{2, k}\right)_{k \geq 0}$ converges uniformly to $\rho_{2}$. Since the measure $\mu_{\infty}$ has finite total mass and, by the previous case, the functions $\rho_{2, k}$ are $\mu_{\infty}$-integrable, we deduce that $\rho_{2}$ is $\mu_{\infty}$-integrable and that

$$
\lim _{k \rightarrow \infty} \int_{\mathcal{B}(\mathbb{C})} \rho_{2, k}(p) d \mu_{\infty}(p)=\int_{\mathcal{B}(\mathbb{C})} \rho_{2}(p) d \mu_{\infty}(p) .
$$

Therefore, using (2.11) for the functions $\rho_{2, k}$ and [CT, Théorème 4.1], we deduce that (2.11) also holds in the case when all the metrics are semipositive.

Consequently, $\rho=\rho_{1}-\rho_{2}$ is $\mu$-integrable, and using (2.8), (2.10), (2.11), the arithmetic Bézout theorem in (2.2), and the inductive hypothesis, we have

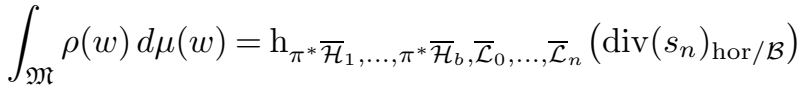

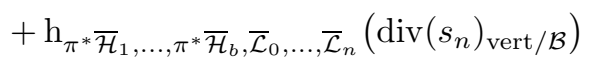

$$
\begin{aligned}
& -\int_{\mathcal{X}(\mathbb{C})} \log \left\|s_{n}\right\|_{n} \bigwedge_{j=0}^{n-1} c_{1}\left(\overline{\mathcal{L}}_{j}\right) \wedge \bigwedge_{i=1}^{b} c_{1}\left(\pi^{*} \overline{\mathcal{H}}_{i}\right)
\end{aligned}
$$

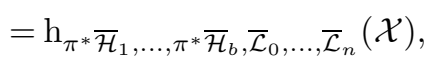

which concludes the proof.

\section{EXAMPLE 2.5}

Let $\mathbb{K} \simeq \mathbb{Q}\left(z_{1}, \ldots, z_{b}\right)$ with the measured set of places $(\mathfrak{M}, \mu)$ as in Examples 1.1 and 1.4. Let

$$
\mathcal{X}=\mathbb{P}_{\mathbb{Z}}^{b} \times \mathbb{P}_{\mathbb{Z}}^{1} \quad \text { and } \quad \overline{\mathcal{L}}=\varpi^{*}{\overline{\mathcal{O}_{\mathbb{P}_{\mathbb{Z}}^{1}}}}^{\text {can }}
$$


where $\varpi$ denotes the projection $\mathbb{P}_{\mathbb{Z}}^{b} \times \mathbb{P}_{\mathbb{Z}}^{1} \rightarrow \mathbb{P}_{\mathbb{Z}}^{1}$, and let $\bar{L}={\overline{\mathcal{O}_{\mathbb{P}_{\mathbb{K}}^{1}}}}^{\text {can }}$ denote the canonical $\mathfrak{M}$-metrized line bundle structure on the universal line bundle of $\mathbb{P}_{\mathbb{K}}^{1}$ as in Example 2.2.

Let $(1: \gamma) \in \mathbb{P}_{\mathbb{Z}}^{1}(\mathbb{K})$ with $\gamma \in \mathbb{K}^{\times}$. The closure $\mathcal{Y}$ of this point in $\mathcal{X}$ is the hypersurface defined by the bihomogenization of the polynomial $P_{\gamma}$ in (1.6). In this case, Theorem 2.4 together with (2.4) and (1.5) gives

$$
\mathrm{h}_{\pi^{*} \overline{\mathcal{H}}_{1}, \ldots, \pi^{*} \overline{\mathcal{H}_{b}}, \overline{\mathcal{L}}}(\mathcal{Y})=\mathrm{h}_{\bar{L}}(1: \gamma)=\mathrm{t}_{\mathbb{K}, \mathfrak{M}, \mu}(\gamma)=\mathrm{m}\left(P_{\gamma}\right),
$$

where $\mathrm{m}\left(P_{\gamma}\right)$ denotes the logarithmic Mahler measure of $P_{\gamma}$.

\section{Height of toric varieties over finitely generated fields}

Using our previous work on toric varieties in [BPS], we can give a combinatorial formula for the mixed height of a toric variety with respect to a family of $\mathfrak{M}$-metrized line bundles. As a consequence of Theorem 2.4, this formula also expresses an arithmetic intersection number, in the sense of Gillet-Soule, of a nontoric arithmetic variety.

Let $\mathcal{B}$ be an arithmetic variety of relative dimension $b$, and $\overline{\mathcal{H}}_{i}=\left(\mathcal{H}_{i},\|\cdot\|_{i}\right)$, $i=1, \ldots, b$, a family of nef Hermitian line bundles on $\mathcal{B}$, as at the beginning of Section 1 . Let $\mathbb{K}=\mathrm{K}(\mathcal{B})$, and let $(\mathfrak{M}, \mu)$ be the associated set of places of $\mathbb{K}$ as in $(1.2)$.

Let $\mathbb{T} \simeq \mathbb{G}_{m}^{n}$ be a split torus of dimension $n$ over $\mathbb{K}$. Let $N=\operatorname{Hom}\left(\mathbb{G}_{m}, \mathbb{T}\right)$ be the lattice of cocharacters of $\mathbb{T}$, let $M=\operatorname{Hom}\left(\mathbb{T}, \mathbb{G}_{m}\right)=N^{\vee}$ be the lattice of characters, set $N_{\mathbb{R}}=N \otimes \mathbb{R}$ and $M_{\mathbb{R}}=M \otimes \mathbb{R}$.

Let $X$ be a proper toric variety over $\mathbb{K}$ with torus $\mathbb{T}$, described by a complete fan $\Sigma$ on $N_{\mathbb{R}}$. A toric divisor on $X$ is a Cartier divisor invariant under the action of $\mathbb{T}$. Such a divisor $D$ defines a virtual support function, that is, a function $\Psi_{D}: N_{\mathbb{R}} \rightarrow \mathbb{R}$ whose restriction to each cone of the fan $\Sigma$ is an element of $M$. The toric divisor $D$ is nef if and only if $\Psi_{D}$ is concave. One can also associate to $D$ the polytope defined as

$$
\Delta_{D}=\left\{x \in M_{\mathbb{R}} \mid x \geq \Psi_{D}\right\} .
$$

Now let

$$
\pi: \mathcal{X} \rightarrow \mathcal{B}
$$

be a dominant morphism of arithmetic varieties of relative dimension $n \geq 0$, and $\overline{\mathcal{L}}$ a Hermitian line bundle on $\mathcal{X}$. We assume that $(X, L)$, the fiber of $(\mathcal{X}, \mathcal{L})$ over the generic point of $\mathcal{B}$, is a toric variety over $\mathbb{K}$ with a line bundle associated to a toric divisor $D$ on $X$. We consider the associated $\mathfrak{M}$-metrized line bundle $\bar{L}$ on $X$ as in (2.1).

For each place $w \in \mathfrak{M}$, we associate to the torus $\mathbb{T}$ an analytic space $\mathbb{T}_{w}^{\text {an }}$ and we denote by $\mathbb{S}_{w}$ its compact subtorus. In the Archimedean case, it is isomorphic to $\left(S^{1}\right)^{n}$. In the non-Archimedean case, it is a compact analytic group (see [BPS, Section 4.2] for a description). Then, the $\mathfrak{M}$-metrized line bundle $\bar{L}=\overline{\mathcal{O}(D)}$ on 
$X$ is toric if its $w$-adic metric $\|\cdot\|_{w}$ is invariant with respect to the action of $\mathbb{S}_{w}$ for all $w$.

Assume that $\bar{L}$ is toric, and let $s$ be the toric section of $L$ with $\operatorname{div}(s)=D$. For each $w \in \mathfrak{M}$, denote by $X_{0, w}^{\text {an }}$ the analytification of the open principal subset $X_{0} \subset X$ corresponding to the cone $\{0\}$, which is isomorphic to the torus $\mathbb{T}$. Then the function $X_{0, w}^{\text {an }} \rightarrow \mathbb{R}$ given by $p \mapsto \log \|s(p)\|_{w}$ is invariant under the action of $\mathbb{S}_{w}$ and induces a function $\psi_{\bar{L}, s, w}: N_{\mathbb{R}} \rightarrow \mathbb{R}$ as in [BPS, Definition 4.3.5]. For shorthand, when $\bar{L}$ and $s$ are fixed, we will denote $\psi_{\bar{L}, s, w}$ by $\psi_{w}$.

We now further assume that the line bundle $\mathcal{L}$ is generated by global sections and that the Hermitian metric on $\overline{\mathcal{L}}$ is semipositive. Hence, the line bundle $L$ is also generated by global sections, and for each $w \in \mathfrak{M}$, the metric induced on $L_{w}^{\text {an }}$ by $\overline{\mathcal{L}}$ is semipositive. In this case, by [BPS, Theorem 4.8.1], for each $w \in \mathfrak{M}$, the function $\psi_{w}$ is concave. We associate to it a concave function on $\Delta_{D}$, denoted by $\vartheta_{\bar{L}, s, w}$ (or $\vartheta_{w}$ for short) and called the $w$-adic roof function of the pair $(\bar{L}, s)$ as in [BPS, Definition 5.1.4]. This function is defined as the Legendre-Fenchel dual of $\psi_{w}$, and so it is defined, for $x \in \Delta_{D}$, as

$$
\vartheta_{w}(x)=\inf _{u \in N_{\mathbb{R}}}\left(\langle x, u\rangle-\psi_{w}(u)\right) .
$$

We denote by $\operatorname{vol}_{M}$ the measure on $\Delta_{D}$ given by the restriction of the Haar measure on $M_{\mathbb{R}}$ normalized so that the lattice $M$ has covolume 1 .

COROLLARY 3.1

With notation as above, the function

$$
\mathfrak{M} \longrightarrow \mathbb{R}, \quad w \longmapsto \int_{\Delta_{D}} \vartheta_{\bar{L}, s, w}(x) d \operatorname{vol}_{M}(x)
$$

is $\mu$-integrable. Moreover,

$$
\begin{aligned}
& \mathrm{h}_{\pi^{*} \overline{\mathcal{H}}_{1}, \ldots, \pi^{*} \overline{\mathcal{H}}_{b}, \overline{\mathcal{L}}, \ldots, \overline{\mathcal{L}}}(\mathcal{X}) \\
& \quad=\mathrm{h}_{\bar{L}}(X)=(n+1) ! \int_{\mathfrak{M}} \int_{\Delta_{D}} \vartheta_{\bar{L}, s, w}(x) d \operatorname{vol}_{M}(x) d \mu(w) .
\end{aligned}
$$

Proof

By [BPS, Theorem 5.1.6], the quantity

$$
(n+1) ! \int_{\Delta_{D}} \vartheta_{\bar{L}, s, w}(x) d \operatorname{vol}_{M}(x)
$$

is equal to the difference of local heights

$$
\begin{aligned}
& \mathrm{h}_{\left(L_{0},\|\cdot\|_{0, w}\right), \ldots,\left(L_{n},\|\cdot\|_{n, w}\right)}\left(Y ; s_{0}, \ldots, s_{n}\right) \\
& \quad-\mathrm{h}_{\left(L_{0},\|\cdot\|_{0, w, \text { can }}\right), \ldots,\left(L_{n},\|\cdot\|_{n, w, \text { can }}\right)}\left(Y ; s_{0}, \ldots, s_{n}\right),
\end{aligned}
$$

where $\|\cdot\|_{i, w \text {,can }}$ is the canonical $w$-adic metric on $L_{i}$ as in [BPS, PropositionDefinition 4.3.15]. By Theorem 2.4, both local heights are $\mu$-integrable. Hence, the function in (3.1) is as well, which proves the first statement. 
For the second statement, the first equality follows from Theorem 2.4. By the discussion above,

$$
(n+1) ! \int_{\mathfrak{M}} \int_{\Delta_{D}} \vartheta_{\bar{L}, s, w}(x) d \operatorname{vol}_{M}(x) d \mu(w)=\mathrm{h}_{\bar{L}}(X)-\mathrm{h}_{\bar{L}} \operatorname{can}(X) .
$$

By using the argument in the proof of [BPS, Proposition 5.2.4], it can be shown that $\mathrm{h}_{\bar{L}^{\text {can }}}(X)=0$, which proves the second equality in (3.2).

\section{THEOREM 3.2}

Let the notation be as above.

(a) For each $x \in \Delta_{D}$, the function

$$
\mathfrak{M} \longrightarrow \mathbb{R}, \quad w \longmapsto \vartheta_{w}(x)
$$

is $\mu$-integrable.

(b) The function

$$
\Delta_{D} \longrightarrow \mathbb{R}, \quad x \longmapsto \int_{\mathfrak{M}} \vartheta_{w}(x) d \mu(w)
$$

is concave and continuous on $\Delta_{D}$.

(c) The function

$$
\mathfrak{M} \times \Delta_{D} \longrightarrow \mathbb{R}, \quad(w, x) \longmapsto \vartheta_{w}(x)
$$

is $\left(\mu \times \operatorname{vol}_{M}\right)$-integrable.

\section{Proof}

Let $\sigma \in \Sigma^{n}$. The closure $V(\sigma)$ of the orbit of $X$ corresponding to $\sigma$ is a point. By [BPS, Proposition 4.8.9], for each $w \in \mathfrak{M}$,

$$
\vartheta_{\iota^{*} \bar{L}, \iota^{*} s_{\sigma}, w}(0)=\vartheta_{\bar{L}, s_{\sigma}, w}\left(m_{\sigma}\right)=\vartheta_{w}\left(m_{\sigma}\right),
$$

where $\iota$ denotes the inclusion $V(\sigma) \hookrightarrow X$. By Corollary 3.1, the function $w \mapsto$ $\vartheta_{w}\left(m_{\sigma}\right)$ is $\mu$-integrable, and its integral coincides with the height of $V(\sigma)$ with respect to $\bar{L}$.

Since $\vartheta_{w}$ is a concave function, for all $x \in \Delta_{D}$,

$$
\min _{\sigma \in \Sigma^{n}} \vartheta_{w}\left(m_{\sigma}\right)=\min _{y \in \Delta_{D}} \vartheta_{w}(y) \leq \vartheta_{w}(x) .
$$

On the other hand, using again the concavity of $\vartheta_{w}$, we have

$$
\begin{aligned}
\vartheta_{w}(x)-\min _{y \in \Delta_{D}} \vartheta_{w}(y) & \leq \max _{y \in \Delta_{D}} \vartheta_{w}(y)-\min _{y \in \Delta_{D}} \vartheta_{w}(y) \\
& \leq \frac{n+1}{\operatorname{vol}_{M}\left(\Delta_{D}\right)} \int_{\Delta_{D}}\left(\vartheta_{w}(z)-\min _{y \in \Delta_{D}} \vartheta_{w}(y)\right) d \operatorname{vol}_{M}(z) .
\end{aligned}
$$

It follows from (3.3) and (3.4) that, for all $x \in \Delta_{D}$,

$$
\min _{\sigma \in \Sigma^{n}} \vartheta_{w}\left(m_{\sigma}\right) \leq \vartheta_{w}(x) \leq \frac{n+1}{\operatorname{vol}_{M}\left(\Delta_{D}\right)} \int_{\Delta_{D}} \vartheta_{w}(z) d \operatorname{vol}_{M}(z)-n \min _{\sigma \in \Sigma^{n}} \vartheta_{w}\left(m_{\sigma}\right) .
$$


By Corollary 3.1 and the fact that $\Delta_{D}$ has finite measure, we have that both the upper and the lower bounds are integrable with respect to the measure $\mu \times \operatorname{vol}_{M}$. The statements (a) and (c) follow directly from these bounds, while the statement (b) follows from the same bounds and Lebesgue's bounded convergence theorem [F, Theorem 2.4.9].

\section{DEFINITION 3.3}

With notation as above, the (global) roof function is the continuous concave function $\vartheta_{\bar{L}, s}: \Delta_{D} \rightarrow \mathbb{R}$ given by

$$
\vartheta_{\bar{L}, s}(x)=\int_{\mathfrak{M}} \vartheta_{w}(x) d \mu(w) .
$$

COROLLARY 3.4

With the previous notation,

$$
\mathrm{h}_{\pi^{*} \overline{\mathcal{H}}_{1}, \ldots, \pi^{*} \overline{\mathcal{H}}_{b}, \overline{\mathcal{L}}, \ldots, \overline{\mathcal{L}}}(\mathcal{X})=\mathrm{h}_{\bar{L}}(X)=(n+1) ! \int_{\Delta_{D}} \vartheta_{\bar{L}, s}(x) d \operatorname{vol}_{M}(x)
$$

holds.

Proof

This follows from Corollary 3.1 and Theorem 3.2(c) together with Fubini's theorem [F, Theorem 2.6.2].

\section{REMARK 3.5}

More generally, when we have a family $\overline{\mathcal{L}}_{0}, \ldots, \overline{\mathcal{L}}_{n}$ of semipositive Hermitian line bundles on $\mathcal{X}$ such that the induced Hermitian line bundles $\bar{L}_{0}, \ldots, \bar{L}_{n}$ on $X$ are toric, we can express

$$
\mathrm{h}_{\pi * \overline{\mathcal{H}}_{1}, \ldots, \pi * \overline{\mathcal{H}}_{b}, \overline{\mathcal{L}}_{0}, \ldots, \overline{\mathcal{L}}_{n}}(\mathcal{X})=\mathrm{h}_{\bar{L}_{0}, \ldots, \bar{L}_{n}}(X)
$$

in terms of mixed integrals, as in [BPS, Theorem 5.2.5].

\section{Canonical height of translates of subtori over finitely generated fields}

In this section, we particularize the formulae in Section 3 to the case when $X$ is the normalization of a translate of a subtorus in the projective space.

As before, let $\mathcal{B}$ be an arithmetic variety of relative dimension $b$, and $\overline{\mathcal{H}}_{i}=$ $\left(\mathcal{H}_{i},\|\cdot\|_{i}\right), i=1, \ldots, b$, a family of nef Hermitian line bundles on $\mathcal{B}$. Let $\mathbb{K}=\mathrm{K}(\mathcal{B})$, and let $(\mathfrak{M}, \mu)$ be the associated set of places of $\mathbb{K}$ as in (1.2).

Let $r \geq 1$, and consider the projective space $\mathbb{P}_{\mathcal{B}}^{r}$ over $\mathcal{B}$ and the universal line bundle $\mathcal{O}_{\mathbb{P}_{\mathcal{B}}^{r}}(1)$ on it. Since $\mathbb{P}_{\mathcal{B}}^{r}=\mathbb{P}_{\mathbb{Z}}^{r} \underset{\operatorname{Spec}(\mathbb{Z})}{\times} \mathcal{B}$ and $\mathcal{O}_{\mathbb{P}_{\mathcal{B}}^{r}}(1)$ is the pullback of $\mathcal{O}_{\mathbb{P}_{\mathbb{Z}}^{r}}(1)$ under the first projection, we can pull back the canonical metric on $\mathcal{O}_{\mathbb{P}_{\mathbb{Z}}^{r}}(1)$ to obtain a metric on $\mathcal{O}_{\mathbb{P}_{\mathcal{B}}^{r}}(1)$, also called canonical. We denote by $\overline{\mathcal{O}(1)}=\overline{\mathcal{O}_{\mathbb{P}_{\mathcal{B}}^{r}}(1)}$ the obtained Hermitian line bundle.

Choose $\mathbf{m}_{j} \in \mathbb{Z}^{n}$ and $f_{j} \in \mathbb{K}^{\times}, j=0, \ldots, r$. For simplicity, we assume that $\mathbf{m}_{0}=0 \in \mathbb{Z}^{n}$ and that the collection of vectors $\mathbf{m}_{j}$ generates $\mathbb{Z}^{n}$ as an abelian 
group. Consider the map

$$
\mathbb{G}_{m, \mathbb{K}}^{n} \longrightarrow \mathbb{P}_{\mathbb{K}}^{r}, \quad \mathbf{t} \longmapsto\left(f_{0} \mathbf{t}^{\mathbf{m}_{0}}: \cdots: f_{r} \mathbf{t}^{\mathbf{m}_{r}}\right),
$$

where $f_{j} \mathbf{t}^{\mathbf{m}_{j}}$ denotes the monomial $f_{j} t_{1}^{m_{j, 1}} \cdots t_{n}^{m_{j, n}}$. We then denote by $Y$ the closure in $\mathbb{P}_{\mathbb{K}}^{r}$ of the image of this map.

The projective space $\mathbb{P}_{\mathbb{K}}^{r}$ is the fiber of $\mathbb{P}_{\mathcal{B}}^{r}$ over the generic point of $\mathcal{B}$. We denote by $\mathcal{Y}$ the closure of $Y$ in $\mathbb{P}_{\mathcal{B}}^{r}$ and by $\pi: \mathcal{Y} \rightarrow \mathcal{B}$ the dominant map obtained by restricting the projection $\mathbb{P}_{\mathcal{B}}^{r} \rightarrow \mathcal{B}$. In this setting, we want to give a formula for the arithmetic intersection number

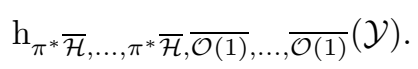

The subvariety $Y$ is not a toric variety over $\mathbb{K}$ because it is not necessarily normal. Indeed, it is a translated toric subvariety of $\mathbb{P}_{\mathbb{K}}^{r}$ in the sense of [BPS, Definition 3.2.6]. Let $\mathcal{X}$ be the normalization of $\mathcal{Y}$, and $X$ the corresponding variety over $\mathbb{K}$. Let $\overline{\mathcal{L}}$ be the pullback of $\overline{\mathcal{O}(1)}$ to $\mathcal{X}$, and $\bar{L}$ the associated $\mathfrak{M}$-metrized line bundle over $X$ as in (2.1). Therefore, $X$ is a toric variety over $\mathbb{K}$ with torus $\mathbb{G}_{m, \mathbb{K}}^{n}$, and the $\mathfrak{M}$-metrized line bundle $\bar{L}$ is toric and semipositive.

In this case we can give an explicit description of the corresponding $w$-adic roof functions.

\section{PROPOSITION 4.1}

With notation as above, let $s$ be the toric section of $L$ determined by the section $x_{0}$ of $\mathcal{O}(1)$. The polytope associated to the divisor $D=\operatorname{div}(s)$ on $X$ is given by

$$
\Delta=\operatorname{conv}\left(\mathbf{m}_{0}, \ldots, \mathbf{m}_{r}\right),
$$

and for $w \in \mathfrak{M}$, the $w$-adic roof function $\vartheta_{w}: \Delta \rightarrow \mathbb{R}$ is the function parameterizing the upper envelope of the extended polytope $\widetilde{\Delta}_{w} \subset \mathbb{R}^{n} \times \mathbb{R}$ given by

$$
\widetilde{\Delta}_{w}= \begin{cases}\operatorname{conv}\left(\left(\mathbf{m}_{j},-\mathrm{h}_{\overline{\mathcal{H}}_{1}, \ldots, \overline{\mathcal{H}}_{b}}(\mathcal{V}) \operatorname{ord} \mathcal{V}\left(f_{j}\right)\right)_{j=0, \ldots, r}\right) & \text { if } w=\mathcal{V} \in \mathcal{B}^{(1)} \\ \operatorname{conv}\left(\left(\mathbf{m}_{j}, \log \left|f_{j}(p)\right|\right)_{j=0, \ldots, r}\right) & \text { if } w=p \in \mathcal{B}(\mathbb{C})^{\text {gen }}\end{cases}
$$

\section{Proof}

This follows from [BPS, Example 5.1.16].

Combining this result with Corollary 3.1, we obtain a formula for the arithmetic intersection number in (4.1), which we will particularize to concrete examples.

\section{COROLLARY 4.2}

With notation as above, set $\mathcal{B}=\mathbb{P}_{\mathbb{Z}}^{b}$ and $\overline{\mathcal{H}}_{i}=\overline{\mathcal{H}}={\overline{\mathcal{O}_{\mathbb{P}_{\mathcal{B}}^{r}}}}^{\text {can }}, i=1, \ldots, b$. Then

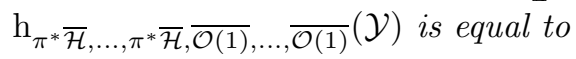

$$
(n+1) !\left(\int\left(\int_{\Delta} \vartheta_{p}(x) d \operatorname{vol}(x)\right) d \mu_{\mathbb{S}}+\sum_{\mathcal{V} \in \mathcal{C}} \int_{\Delta} \vartheta_{\mathcal{V}}(x) d \operatorname{vol}(x)\right),
$$


where $\mathcal{C} \subset \mathcal{B}^{(1)}$ is the set of irreducible components of the divisors $\operatorname{div}\left(f_{j}\right), j=$ $0, \ldots, r$, vol denotes the Lebesgue measure on $\mathbb{R}^{n}$, and $\mu_{\mathbb{S}}$ is the Haar measure of the compact torus $\mathbb{S}$ as in Example 1.1.

Proof

We have that $\mathrm{h}_{\pi * \overline{\mathcal{H}}}, \ldots, \pi * \overline{\mathcal{H}}, \overline{\mathcal{O}(1)}, \ldots, \overline{\mathcal{O}(1)}(\mathcal{Y})=\mathrm{h}_{\pi^{*} \overline{\mathcal{H}}, \ldots, \pi^{*} \overline{\mathcal{H}}, \overline{\mathcal{L}}, \ldots, \overline{\mathcal{L}}}(\mathcal{X})$ by the invariance of the height under normalization. The formula then follows from Corollary 3.1, Proposition 4.1, the description of the measured set of places $(\mathfrak{M}, \mu)$ of the field $\mathbb{K}$ in Example 1.1, and the fact that, for $\mathcal{V} \in \mathcal{B}^{(1)} \backslash \mathcal{C}$, the local roof function $\vartheta_{\mathcal{V}}$ vanishes identically.

\section{EXAMPLE 4.3}

Consider the case $n=0$. As a result, $\mathbf{m}_{\ell}=0$ for all $\ell$. Choose a collection $f_{0}, \ldots, f_{r} \in \mathbb{Z}\left[z_{1}, \ldots, z_{b}\right]$ of coprime polynomials with integer coefficients. Hence, $\Delta=\{0\} \subset \mathbb{R}^{0}$, and for $w \in \mathfrak{M}$, the local roof function is given by

$$
\vartheta_{w}(0)= \begin{cases}\max _{\ell} \log \left|f_{\ell}(p)\right| & \text { if } w=p \in \mathbb{P}_{\mathbb{Z}}^{b}(\mathbb{C})^{\text {gen }}, \\ -\mathrm{h}(\mathcal{V}) \min _{\ell} \operatorname{ord}_{\mathcal{V}}\left(f_{\ell}\right) & \text { if } w=\mathcal{V} \in\left(\mathbb{P}_{\mathbb{Z}}^{b}\right)^{(1)} .\end{cases}
$$

If $\mathcal{V}$ is not the hyperplane at infinity of $\mathbb{P}^{b}$, then by the coprimality of the $f_{\ell}$ 's, we have $\min _{\ell}$ ord $\mathcal{V}\left(f_{\ell}\right)=0$, while if $\mathcal{V}$ is the hyperplane at infinity, then $\mathrm{h}(\mathcal{V})=0$. Thus the finite contribution vanishes, and from Theorem 2.4 and Proposition 4.1, we deduce

$$
\mathrm{h}_{\pi^{*} \overline{\mathcal{H}}, \ldots, \pi^{*} \overline{\mathcal{H}}, \overline{\mathcal{L}}}(\mathcal{Y})=\int \max _{\ell}\left(\log \left|f_{\ell}(p)\right|\right) d \mu_{\mathbb{S}}
$$

In particular, if $r=1$, then this arithmetic intersection number agrees with the size of the element $\gamma=f_{1} / f_{0}$ given in Example 1.4. For instance, consider the case when $b=1, f_{0}=\alpha$, and $f_{1}=\beta z_{1}+\gamma$ with $\alpha, \beta, \gamma$ coprime integers. By using (1.5) and (1.6), the corresponding intersection number is given by the logarithmic Mahler measure of the affine polynomial $\alpha t_{1}-\beta z_{1}-\gamma$. By [Ma, Proposition 7.3.1], this logarithmic Mahler measure can be computed in terms of the Bloch-Wigner dilogarithm.

\section{EXAMPLE 4.4}

Let $n=1$, and consider the case when $m_{i}=i, i=0, \ldots, r$, and $f_{0}, \ldots, f_{r} \in$ $\mathbb{Z}\left[z_{1}, \ldots, z_{b}\right]$ is a family of coprime polynomials with integer coefficients with $f_{0}=f_{r}=1$. We have that $\Delta=[0, r]$. Let $w \in \mathfrak{M}$, and let $\vartheta_{w}:[0, r] \rightarrow \mathbb{R}$ be the corresponding local roof function. By Proposition 4.1, if $w=\mathcal{V} \in\left(\mathbb{P}_{\mathbb{Z}}^{b}\right)^{(1)}$, then this function is zero. Also, if $w=p \in \mathbb{P}_{\mathbb{Z}}^{b}(\mathbb{C})^{\text {gen }}$, then this function is the minimal concave function on $[0, r]$ whose values at the integers are given, for $i=0, \ldots, r$, by

$$
\vartheta_{w}(i)=\max _{\substack{0 \leq j \leq i \leq \ell \leq r \\ j \neq \ell}}\left(\frac{\ell-i}{\ell-j} \log \left|f_{j}(p)\right|_{w}+\frac{i-j}{\ell-j} \log \left|f_{\ell}(p)\right|_{w}\right) .
$$


In particular, $\vartheta_{w}(0)=\log \left|f_{0}(p)\right|_{w}=0$ and $\vartheta_{w}(r)=\log \left|f_{r}(p)\right|_{w}=0$. It follows that

$$
\int_{0}^{r} \vartheta_{w}(x) d x=\sum_{i=1}^{\delta-1} \max _{j \leq i \leq \ell, j \neq \ell}\left(\frac{\ell-i}{\ell-j} \log \left|f_{j}(p)\right|+\frac{i-j}{\ell-j} \log \left|f_{\ell}(p)\right|\right) .
$$

From Corollary 4.2 and (4.2), we deduce that

$$
\mathrm{h}_{\pi^{*} \overline{\mathcal{H}}, \ldots, \pi^{*} \overline{\mathcal{H}}, \overline{\mathcal{L}}}(\mathcal{Y})=2 \int \sum_{i=1}^{r-1} \max _{\substack{0 \leq j \leq i \leq \ell \leq r \\ j \neq \ell}}\left(\frac{\ell-i}{\ell-j} \log \left|f_{j}(p)\right|+\frac{i-j}{\ell-j} \log \left|f_{j}(p)\right|\right) d \mu_{\mathbb{S}} .
$$

Hence, this arithmetic intersection number can be expressed in terms of integrals over the compact torus, of maxima of logarithms of absolute values of polynomials.

Acknowledgments. Part of this work was done while the authors met at the Universitat de Barcelona, the Instituto de Ciencias Matemáticas (Madrid), and the Institut de Mathématiques de Jussieu (Paris). We are thankful to these institutions for their hospitality. We also thank Julius Hertel and the referee for their useful comments.

\section{References}

[B] P. Billingsley, Convergence of Probability Measures, 2nd ed., Wiley, New York, 1999. MR 1700749. DOI 10.1002/9780470316962.

[BGS] J.-B. Bost, H. Gillet, and C. Soulé, Heights of projective varieties and positive Green forms, J. Amer. Math. Soc. 7 (1994), 903-1027. MR 1260106. DOI 10.2307/2152736.

[BMPS] J. I. Burgos Gil, A. Moriwaki, P. Philippon, and M. Sombra, Arithmetic positivity on toric varieties, to appear in J. Algebraic Geom., preprint, arXiv:1210.7692v1 [math.AG].

[BPS] J. I. Burgos Gil, P. Philippon, and M. Sombra, Arithmetic Geometry of Toric Varieties: Metrics, Measures and Heights, Astérisque 360, Soc. Math. France, Paris, 2014. MR 3222615.

[CT] A. Chambert-Loir and A. Thuillier, Mesures de Mahler et équidistribution logarithmique, Ann. Inst. Fourier (Grenoble) 59 (2009), 977-1014. MR 2543659.

[F] H. Federer, Geometric Measure Theory, Grundlehren Math. Wiss. 153, Springer, New York, 1969. MR 0257325.

[G] W. Gubler, Local and canonical heights of subvarieties, Ann. Sci. Norm. Sup. Pisa Cl. Sci. (5) 2 (2003), 711-760. MR 2040641.

[Ma] V. Maillot, Géométrie d'Arakelov des variétés toriques et fibrés en droites intégrables, Mém. Soc. Math. Fr. (N.S.) 80, Soc. Math. France, Paris, 2000. MR 1775582. 
[Mo1] A. Moriwaki, Arithmetic height functions over finitely generated fields, Invent. Math. 140 (2000), 101-142. MR 1779799. DOI $10.1007 / \mathrm{s} 002220050358$.

[Mo2] The canonical arithmetic height of subvarieties of an abelian variety over a finitely generated field, J. Reine Angew. Math. 530 (2001), 33-54. MR 1807267. DOI 10.1515/crll.2001.005.

[Mo3] A generalization of conjectures of Bogomolov and Lang over finitely generated fields, Duke Math. J. 107 (2001), 85-102. MR 1815251. DOI 10.1215/S0012-7094-01-10715-1.

[YZ] X. Yuan and S.-W. Zhang, The arithmetic Hodge index theorem for adelic line bundles, II: Finitely generated fields, preprint, arXiv:1304.3539v1 [math.NT].

[Z] S. Zhang, Small points and adelic metrics, J. Algebraic Geom. 4 (1995), 281-300. MR 1311351.

Burgos Gil: Instituto de Ciencias Matemáticas (CSIC), Madrid, Spain;

burgos@icmat.es; http://www.icmat.es/miembros/burgos

Philippon: Équipe de Théorie des Nombres, Institut de Mathématiques de Jussieu, UMR CNRS 7586, Paris, France; patrice.philippon@imj-prg.fr;

http://www.math.jussieu.fr/ pph

Sombra: Departament d'Àlgebra i Geometria, Universitat de Barcelona, and Catalan Institution for Research and Advanced Studies (ICREA), Barcelona, Spain; sombra@ub.edu; http://atlas.mat.ub.es/personals/sombra 